\title{
Guerre civile et résilience écologique : le cas du Parc national du mont Sangbé à l'ouest de la Côte d'Ivoire*
}

Thomas Bearth $^{1}$ Joseph Baya ${ }^{2}$

${ }^{1}$ Department of General Linguistics University of Zurich

Langäckerstrasse 28

$\mathrm{CH}$-8957 Spreitenbach

Switzerland

<Thomas_Bearth@compuserve.com>

2 Projet LAGSUS

(Language, Gender and Sustainability) c/o Centre suisse de recherches scientifiques en Côte d'Ivoire

01 BP 1303

Abidjan-01

Côte d'Ivoire

<bayajoseph@yahoo.fr>

\section{Résumé}

Bénéfice écologique pour le reste du monde, perte économique pour les riverains : le premier bilan de l'aménagement du Parc national du Mont Sangbé, situé dans la zone limitrophe entre savane et forêt dans l'Ouest de la Côte d'Ivoire, ne laissait guère présager l'adhésion de la population riveraine du parc aux enjeux de sa conservation, encore moins au moment où, dans le sillage de la guerre civile, les pillages à main armée menacent d'extinction la biodiversité du parc et où cette même population se voit privée de ses meilleurs atouts économiques, dont les retombées escomptées de sa valorisation à des fins touristiques. L'élucidation de ce paradoxe tient compte de l'analyse faite par les acteurs eux-mêmes et fait ressortir le lien entre une stratégie de sensibilisation privilégiant les ressources communicationnelles locales et la genèse d'une motivation écologique endogène.

Mots clés : conflit armé ; Côte d'Ivoire ; dégât ; développement durable.

Thèmes : économie et développement rural ; forêts ; ressources naturelles et environnement.

\section{Abstract \\ Civil war and ecological resilience: The case of the Mont Sangbe National Park in Western Côte d'ivoire}

The paper reports on participatory research carried out from 2004 to 2008 in rebel-held Western Côte d'Ivoire. Its focus is on discourses of ownership and resilience among local populations in the peripheral zone of the EU-sponsored Mont Sangbe National Park, the very existence of which is threatened in the wake of a cycle of violence, poverty and unchecked exploitation of remaining natural reserves. Evidence taking into account analysis of this situation by local actors substantiates the claim that, in addition to economic incentives, communicative strategies, scope of inclusion in negotiation, and use of local language as the default medium are decisive for fostering ecologically motivated ownership on which local resilience and post-conflict reconstruction can build.

Key words: armed conflicts; damage; Ivory Coast; sustainable development.

Subjects: economy and rural development; forestry; natural resources and environment. 10.1684/agr.2010.0400 


\section{Objectif et méthode de l'enquête}

Parmi les effets collatéraux les plus destructeurs des rébellions et des guerres figurent les coups portés aux fondements mêmes sur lesquels repose l'espoir de retour à une vie " normale " pour les populations concernées. Les stigmates laissés dans le milieu naturel par un conflit ont souvent des conséquences dramatiques pour ces dernières : démunies et appauvries par l'effondrement d'une économie déjà fragile en temps normal, elles se voient de surcroît privées des ressources naturelles nécessaires au développement social et individuel au-delà d'une survie au quotidien dans un après-guerre sombre et dépourvu de toute perspective. Des régions entières sont ainsi dévalorisées et l'avenir des générations futures compromis. Mais, contrairement aux dégâts environnementaux causés par les conflits armés (Alvarez, 2003; Price, 2003 ; Halle et Bruzon, 2006), les réactions des populations concernées face à ce type de menace n'ont guère fait l'objet d'enquêtes approfondies, encore moins les ressorts d'une possible résilience. La présente étude de cas est fondée sur l'observation participative d'une population réduite à la précarité extrême par la guerre civile ivoirienne, condition aggravée et prolongée par son enclavement géographique en zone montagneuse. L'étude rend compte d'une résilience manifestée au sein de cette population à l'égard d'un enjeu de conservation qui lui avait été imposé de l'extérieur, à savoir le Parc national du mont Sangbé (PNMS), situé sur son territoire en zone ex-rebelle à l'ouest du pays. Menée de 2003 à 2008 par des enquêteurs résidents, dans le cadre d'une recherche mandatée par la Fondation Volkswagen et bénéficiant de l'appui logistique du Centre suisse de recherches scientifiques en Côte d'Ivoire, l'étude s'inspire d'une hypothèse développée dans le cadre du projet LAGSUS ${ }^{1}$ (Language, Gender and Sustainability), à savoir qu'il existe un lien étroit entre le recours aux langues locales comme moyen d'appropriation des innovations et leur durabilité, hypothèse récapitulée dans le théorème de la durabilité communicationnelle (LAGSUS, 2009). Grâce au contact ininterrompu

\footnotetext{
${ }^{1}$ LAGSUS : Description du projet, fondations,
} résultats (2009) : www.lagsus.de/français avec la population concernée et à l'échange par internet avec des chercheurs externes, l'équipe sur place a pu suivre de près le processus de renégociation endogène des enjeux du parc avec le concours des habitants de sa zone riveraine. Ceux-ci furent associés aux activités de recherche, tantôt comme source principale d'une analyse locale de la crise et de ses effets sur leur propre situation et celle du parc, tantôt comme source de validation des hypothèses et analyses dégagées des discours enregistrés au cours de l'enquête.

\section{Le parc et sa périphérie dans le contexte de la crise ivoirienne}

Forêt classée depuis 1945, déclaré parc national par décret du 19 février 1976 et aménagé de 1995 à 2001 sous l'égide de la Commission européenne et de la République de Côte d'Ivoire, avec un financement de lancement de trois millions d'euros, le PNMS est situé dans la région de Man, en zone de transition forêtsavane, dans l'Ouest du pays (figure 1).

Confiné dans le triangle BiankoumaTouba-Sifié, traversé d'ouest en est par le Bafing, tributaire du Sassandra, le PNMS contribue à un réseau hydrographique qui alimente la vaste étendue du plateau ivoirien, drainant sur son passage vers l'Atlantique la partie occidentale de la "boucle du cacao". Faisant partie des patrimoines naturels et forestiers de la Côte d'Ivoire, le PNMS occupe, avec ses 95000 hectares, la quatrième place après les parcs de Bouna, Taï et Marahoué. Quarante pourcents de cette superficie se situe en zone montagneuse: le massif du mont Sangbé, dernier rebondissement de la dorsale guinéenne vers l'est, culmine à 1052 mètres. Un recensement de la faune en cours, interrompu par la crise, a identifié 69 espèces de mammifères, 12 de reptiles et 60 d'oiseaux (MEEF, 2000).

Les populations riveraines du PNMS, estimées à 47000 personnes, dont environ 750 " déguerpis " (MEEF, 2000), sont circonscrites dans un périmètre appelé "zone périphérique du PNMS". Cette zone, d'une superficie de 200000 hectares comprise dans un rayon de 20 à 40 kilomètres autour du parc, héberge quatre groupes ethniques: les Yacouba au nord-ouest de Biankouma, préfecture à 35 kilomètres au nord de Man; les Toura dans la zone montagneuse au nord-est de Biankouma ; les Mahou dans le département de Touba, à 130 kilomètres au nord de Man, et les Worodougou à Sifié, à 100 kilomètres à l'est de Man (figure 2).

Cette zone se caractérise par une économie de subsistance de type traditionnel fondée sur la culture du riz, du manioc, de l'igname, du gombo, de la patate, du piment et de l'aubergine. En temps normal, l'excédent de production de ces cultures est vendu sur les marchés locaux et régionaux.

L'aménagement du PNMS, effectué dans les années précédant la guerre civile, $s$ 'inscrit dans le projet mondial de conservation et de gestion durable des ressources naturelles. Le fonctionnement du PNMS est de type intégratif (Guéneau et Jacobée, 2005) : réserve naturelle ouverte aux visiteurs et chercheurs, mais dont l'exploitation est interdite aux populations locales; en guise de compensation, ces dernières bénéficient de subventions spéciales attribuées au développement de la zone périphérique du parc ainsi que d'emplois créés par sa mise en valeur touristique.

Jusqu'au déclenchement des hostilités dans la région, l'administration chargée de mener à terme l'aménagement comprenait :

- la cellule administrative responsable de la coordination des activités relatives au parc ;

- la "Mission mont Sangbé " composée de spécialistes des parcs, dont un représentant du Parc naturel régional du Haut Jura, et d'une équipe de l'Association française des volontaires du progrès (AFVP) chargée de la surveillance du développement de la zone périphérique ; - les Comités de développement intervillageois (Codiv), structures locales formées dans le but de relayer à terme l'appareil administratif du parc afin d'en assurer la gestion durable. Les CODIV, composés de deux représentants élus par la population de chacun des 137 villages de la zone périphérique, sont regroupés en dix "pays ruraux" et pilotés par la Fédération des Codiv (Fedeco), habilitée à les représenter devant l'administration. Le rapport final de l'AFVP (Ouattara et al., 2001) tire un bilan positif des efforts faits pour créer, au travers de ces structures locales, un engagement tangible des populations en faveur du parc: "Les deux principaux objectifs relatifs au 


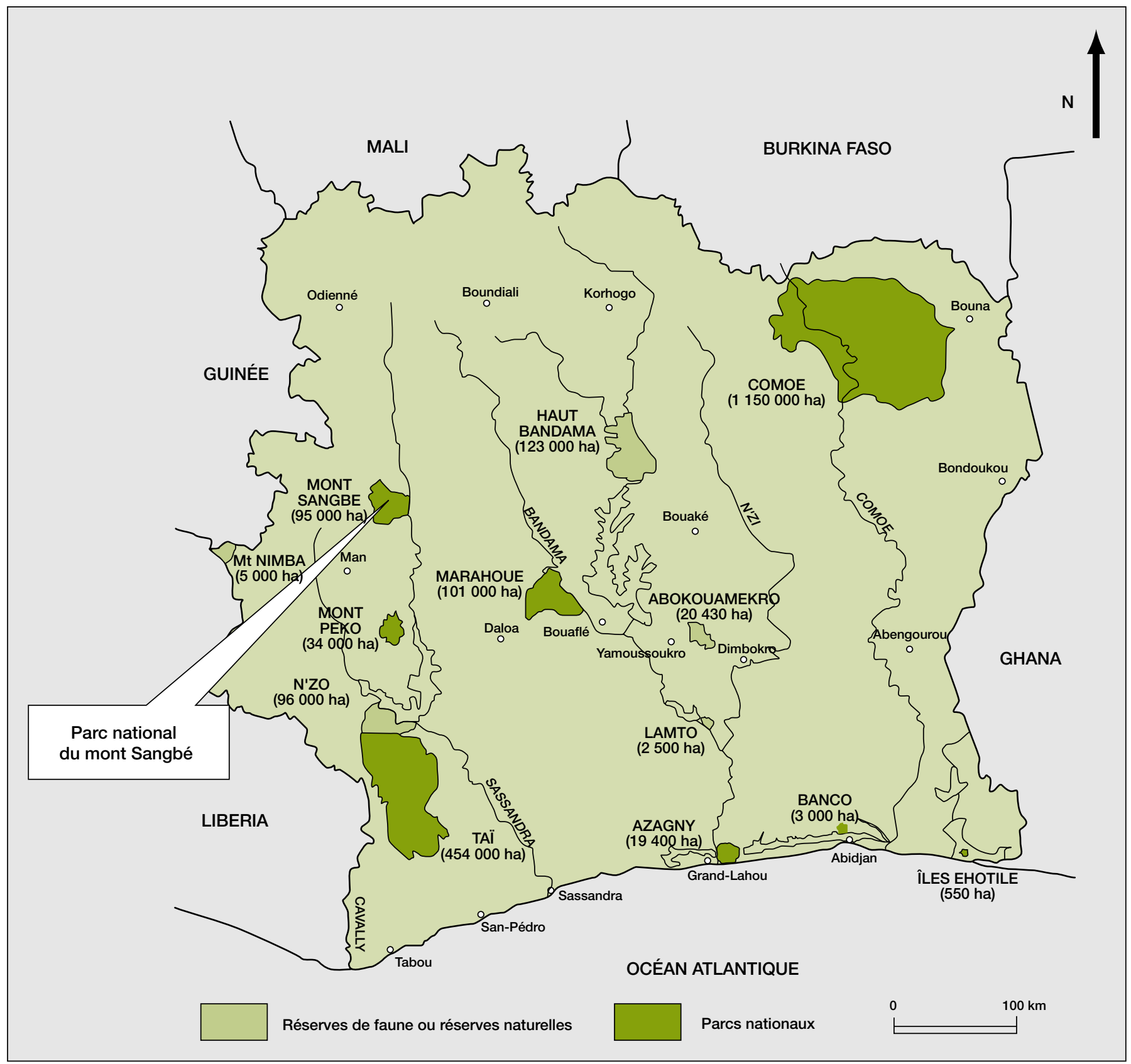

Figure 1. Emplacement géographique du Parc du mont Sangbé.

Figure 1. Geographical location of the PNMS. Source : MEEF, 2000.

développement socio-économique de la zone périphérique et l'implication de la population dans la gestion du Parc National du Mont Sangbé ont été honorés." Toutefois, à la lumière des témoignages recueillis auprès de plusieurs communautés toura, cette appréciation, qui semble suggérer une adhésion générale de la population à l'idéologie du parc et aux principes de sa gestion, appelle quelques réserves.
Ainsi à Kokealo, localité au nord du territoire toura fondée pour accueillir les "déguerpis" du parc, l'attitude contestataire de la population par rapport à la nouvelle vocation de leur terre est résumée par un notable dans une formule succincte : "Ko-à poon' à gá le " 'C'est notre chose.' (c'est-à-dire, le parc nous appartient). Se réalignant aussitôt sur le discours officiel, le locuteur reprend sa phrase pour corriger son " erreur " : "Ko-à poon' nuu à gá le " 'C'était notre chose.' ... Calcul ou lapsus freudien? La transformation de la terre en parc s'assimile pour les gens de Kokealo à un acte de piraterie attribué à kwí (le Blanc), vocable qui désigne tout pouvoir externe interférant avec l'ordre traditionnel sans être légitimé par ce dernier. "Kwíà poon " (chose du Blanc), formule appliquée au parc au moment de sa mise en place, exprime la frustration et l'incompréhension générales 


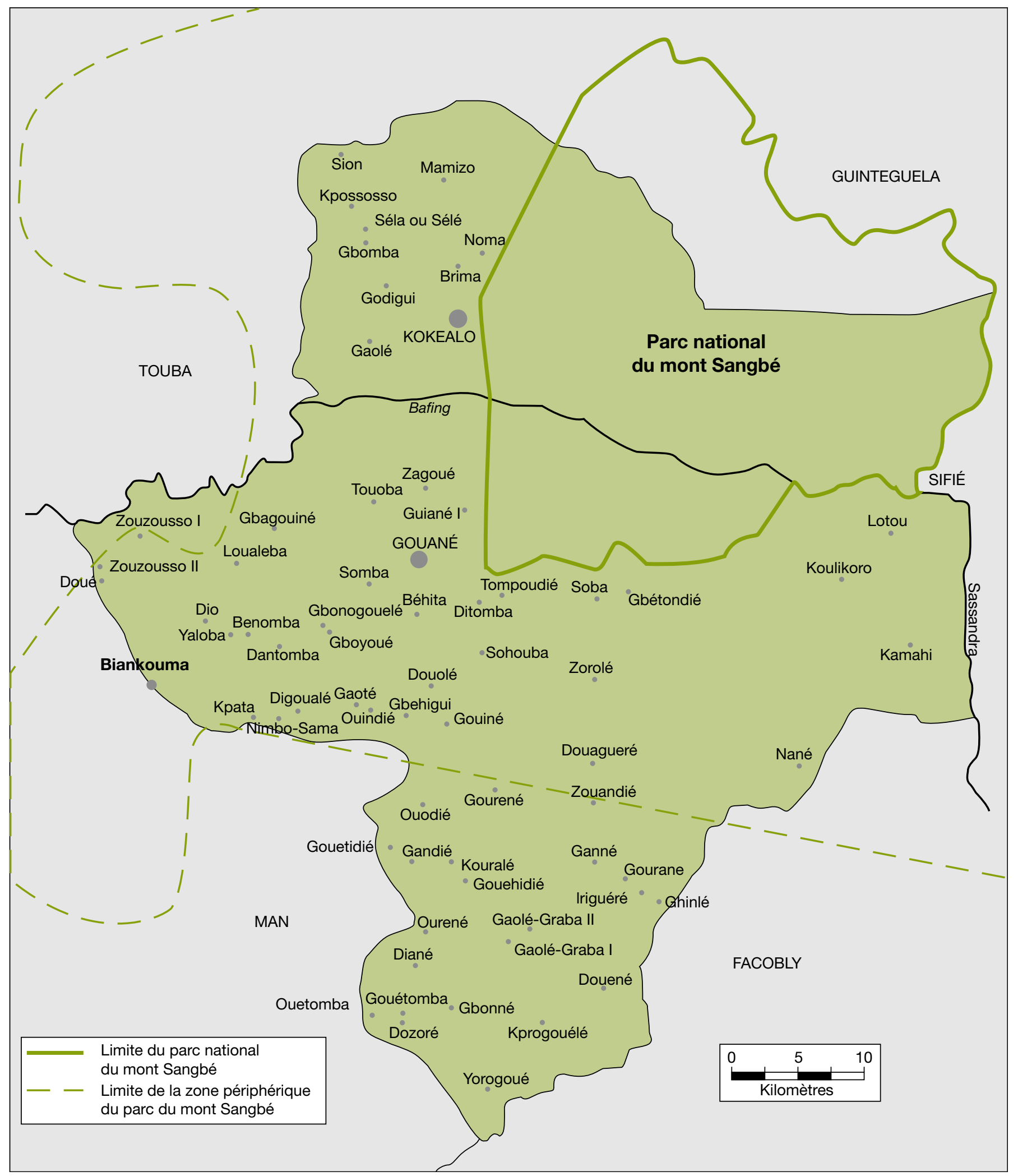

Figure 2. Parc national du mont Sangbé et zone périphérique.

Figure 2. The PNMS and its peripheral zone.

(c) M. Soumahoro, Département de Géographie, Université Laurentienne (Canada), 2009. 
de la population (Baya, 2008) face à l'aliénation de leurs droits ancestraux, dont ceux d'y cultiver la terre, d'y chasser et d'y habiter, sans parler des droits héréditaires des particuliers dont la liste reste à faire.

Si la formule dans ses variations atteste de la part des gens la reconnaissance d'un état de fait qu'ils opposent à un état de droit toujours en vigueur à leurs yeux, ce stade de contestation sournoise semble dépassé lorsque, en février 2005, en pleine crise politico-militaire, le chef du village de Gouané, chef-lieu du pays rural de même nom, la reprend à son compte. Bien que littéralement identique à celle du notable cité plus haut, elle sert à exprimer non pas la revendication d'un bien perdu, mais la réappropriation de ce même bien pour le compte de sa vocation écologique et touristique, dont on est désormais cogestionnaire; on traduirait : "Le parc est notre affaire. "Pour expliquer ce retournement sémantique, nous en proposerons une double lecture en tant que : - réponse d'une communauté rurale au défi moral et économique de la crise qui met en cause son avenir ;

- résultat d'une stratégie d'implication des populations fondée sur les ressources communicationnelles locales et l'autonomie de la gestion.

\section{Séquelles écologiques et économiques de la guerre}

L'irruption de la guerre en novembre 2002 entraîne l'effondrement de l'économie rurale et de toute activité de développement. La chute dramatique des prix du café, du cacao, du coton et du riz contraint les paysans à abandonner les cultures de rente pour ne les reprendre timidement qu'à partir de 2006. Le déclin brutal de l'économie locale, la détérioration des conditions de production, l'impraticabilité des voies d'écoulement et l'accueil de nombreux réfugiés dans sa zone montagneuse - moins touchée par les hostilités - finissent par réduire la population toura à un état de pauvreté sans précédent (Bearth, 2008). Le passage de la région sous le contrôle du pouvoir militaire rebelle ne réussit que très partiellement à combler le vide administratif engendré par le départ des services gouvernementaux. En contrepartie, cet

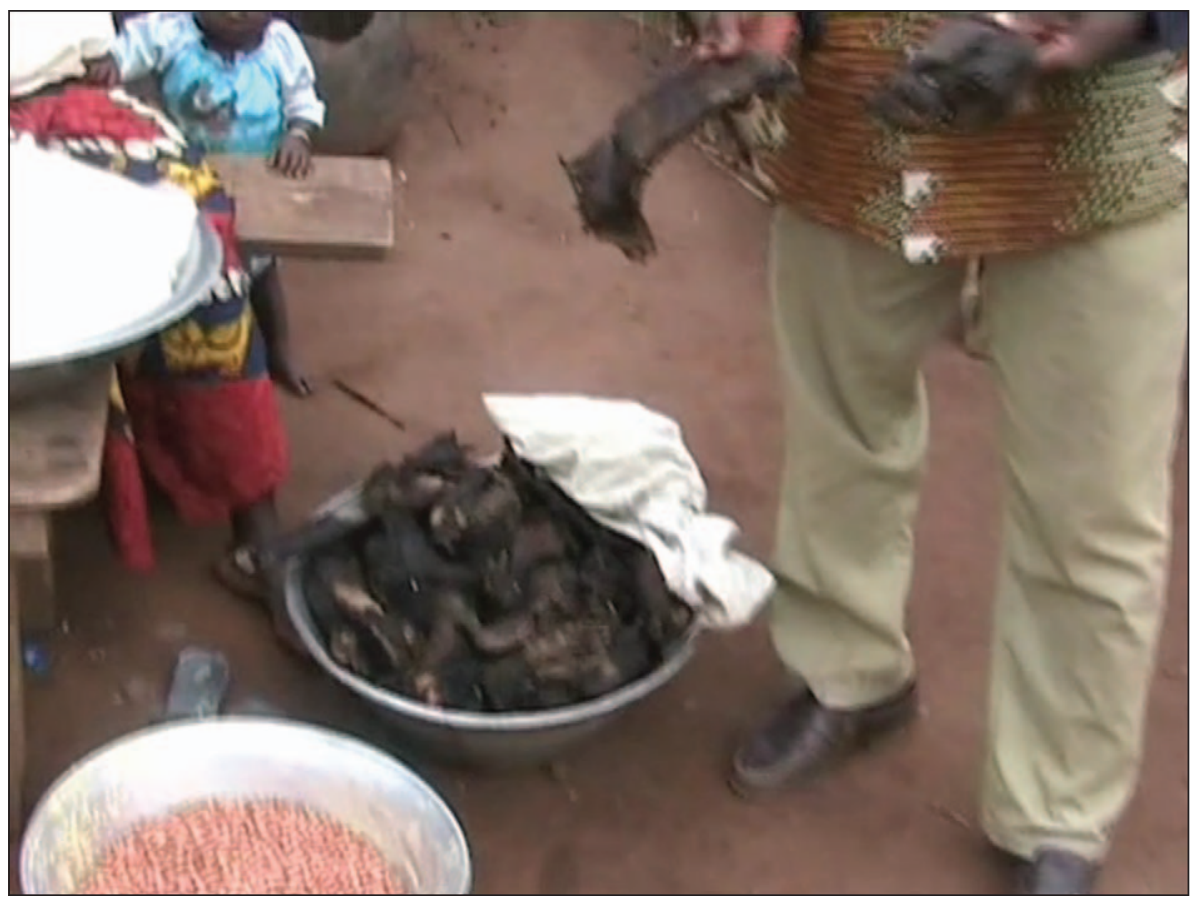

Figure 3. Gibier en provenance du Parc national du mont Sangbé au marché de Dioma.

Figure 3. Meat from the PNMS on a local market.

état de dérèglement et de dénuement favorise l'essor d'une économie prédatrice au détriment de l'environnement et des paysans. Ces derniers, démunis et contraints d'assurer le minimum de revenu nécessaire à leur survie, en sont à la fois victimes et complices : pillage du patrimoine forestier ; vente à vil prix des propriétés terriennes à des exploitants allochtones; commerce de balais à feuilles de palmier pratiqué par les femmes - marché de dupes qui, pour un "gain" de 100 à 200 francs CFA ${ }^{2}$ par palmier sacrifié, risque d'anéantir les palmeraies, une richesse naturelle des monts Toura.

Dès le début des hostilités, les administrateurs du parc et les agents chargés de sa surveillance, à l'instar des autres fonctionnaires, se réfugient dans la zone gouvernementale. Les Codiv, seuls défenseurs attitrés du parc restés sur place, deviennent, après la disparition de l'autorité administrative dont ils tiennent leur mandat, les cibles de menaces d'élimination physique, accusés d'être de mèche avec le pouvoir loyaliste. Cette stratégie fomentée afin de neutraliser leur capacité d'intervention en faveur de la sauvegarde du parc livre ce dernier à la merci des

$\overline{21 \text { euro }}=655,96$ francs CFA. braconniers qui, munis d'armes à feu automatiques, proviennent de partout, voire même des pays limitrophes, pour piller le PNMS. Les marchés locaux regorgent de gibier abattu dans l'enceinte du parc, dont une partie est ensuite revendue en ville (figure 3).

Quelle fut l'attitude de la population face à ce qu'elle perçoit elle-même comme une liquidation de la substance vive du parc? Les témoignages recueillis à partir de janvier 2004 autorisent une double lecture, dans laquelle se mêlent le souci causé par la destruction du patrimoine en tant que valeur à conserver et à transmettre aux générations futures, et le "rasle-bol " d'être, une fois de plus, les grands perdants d'une économie sauvage entretenue par des acteurs venus d'ailleurs qui, du fait de la supériorité de leurs armes, s'enrichissent à leurs dépens. Rien d'étonnant alors à ce que certains villageois tentent leur chance à leur tour, comparant le gain qu'ils tirent de la chasse illicite au rendement désormais ridicule des cultures de rente. "Par jour dix jeunes en moyenne "traversent le village de Dio sur l'axe d'accès qui relie Biankouma à Gouané pour se rendre à la chasse illicite au parc. À défaut de statistiques, cette observation faite lors d'une interview menée en janvier 2004 laisse deviner l'ampleur du phénomène. 


\section{Un tollé}

\section{en faveur du parc}

C'est sur ce fond de pessimisme et de pragmatisme qu'on appréciera à sa juste valeur le regain d'intérêt collectif pour le parc parmi la population locale, notamment dans la zone de Gouané. Coupée du monde extérieur, cette zone ne dispose ni d'électricité ni de réseau téléphonique ni-depuis que la guerre a commencé-de pistes d'accès praticables. L'évolution des mentalités à propos du PNMS peut être retracée au travers des débats publics du Codiv local qui, composé d'une cinquantaine de délégués et de surveillants de 12 localités représentant une population de 2500 habitants, se réunit dans l'enceinte de l'école, désertée après l'exode des instituteurs d'État.

"Lave-toi toi-même le ventre pendant qu'on te lave le dos": ce proverbe, prononcé le 6 février 2006 lors de la relance des activités du Codiv, invite la population démoralisée par la guerre et ses séquelles à reprendre son destin en main, dont les enjeux majeurs sont le parc et le développement de sa périphérie. À l'attitude de victimes résignées face à la crise politico-militaire et à la destruction du parc, l'image du proverbe oppose celle d'acteurs qui, en s'engageant pour le parc sans attendre le retour de son administration, devancent par leur action le dénouement de la crise. Si cet engagement reste limité, par manque des moyens appropriés pour pouvoir s'opposer efficacement au braconnage organisé, le ralliement de la population, sous l'égide des chefs et des notables, autour de la cause du parc, pour contrer sa mise à sac, est en soi un accomplissement majeur, qui sera d'ailleurs reconnu par l'administrateur désigné de la section Ouest du parc lors d'une prise de contact avec les habitants, le 8 juillet 2009, en anticipation de sa relance officielle.

Ce tollé en faveur d'un enjeu écologique menacé, venant d'une population ellemême dénuée de tous ses atouts économiques suite à la guerre, ne s'explique que si l'on tient compte d'un processus d'appropriation ayant déjà abouti bien en amont de celle-ci. Comme le rappelle un notable lors d'une rencontre publique en 2008, l'autonomie accordée par la régie du parc au Codiv de Gouané dès 2001, l'autorisant à gérer l'attribution et à assurer le suivi des subventions allouées au développement de la zone périphérique, avait convaincu la majorité locale: "Eux- mêmes l'ont alors affirmé: "Le parc est notre chose." L'autonomie en matière de gestion n'est pourtant pas la raison de l'engagement local en faveur du parc ; elle en est plutôt la récompense, au même titre qu'une prime attribuée en 2001 au CodiV de Gouané pour avoir mis sur pied un comité local de surveillance du parc qui avait contribué à la baisse marquée du braconnage dans la zone sous son contrôle. Mais en même temps, elle renforce au sein de la population la crédibilité de ceux qui s'étaient laissés convaincre par l'argument écologique.

Détail significatif, le notable qui, en avocat éloquent du parc, retrace ainsi l'évolution des mentalités vers un consensus en sa faveur, n'appartient ni par son âge ni par son éducation au groupe d'interlocuteurs privilégiés par la stratégie de sensibilisation originalement préconisée. Cette dernière devait s'appuyer de préférence sur les jeunes natifs des localités riveraines du parc qui, scolarisés et ayant séjourné en ville, "sont ouverts aux innovations technologiques et doivent devenir, pour le projet, des interlocuteurs de choix" (Engref, 1996). Elle avait ainsi privilégié comme vecteur du message innovateur le français, langue officielle mais peu maîtrisée par la population et ses dignitaires, et comme promoteurs du parc au sein de celle-ci un groupe sommairement assimilé aux "Blancs", dépourvu de statut dans la hiérarchie sociale de la communauté-cible.

Au vu de l'échec manifeste de cette stratégie, l'AFVP admet, à titre expérimental, mais en laissant aux animateurs le choix de l'adopter ou non, une stratégie de sensibilisation inclusive, privilégiant la langue locale comme source de conceptualisation, de réflexion et de négociation, au lieu de la reléguer au statut de moyen de retransmission d'un discours conçu en français. Le parc sera "expliqué " et ses enjeux soumis à la délibération de l'ensemble des habitants de chaque localité au travers du Kono - procédure de consultation publique, fermement ancrée dans la culture locale, qui, par le truchement d'un protocole réglant l'attribution de la parole, met obligatoirement à contribution, avant toute prise de décision, les membres de la communauté concernée par le message, du plus jeune au plus vieux, en réservant le mot décisif aux aînés (Bearth et Fan, 2006) (figure 4). Cette stratégie, en soumettant au contrôle local la gestion des ressources communicationnelles mises en ouvre en vue de la sensibilisation de la population, favorisait la compréhension par celle-ci des enjeux écologiques du parc, au lieu de miser uniquement sur la compensation écono-

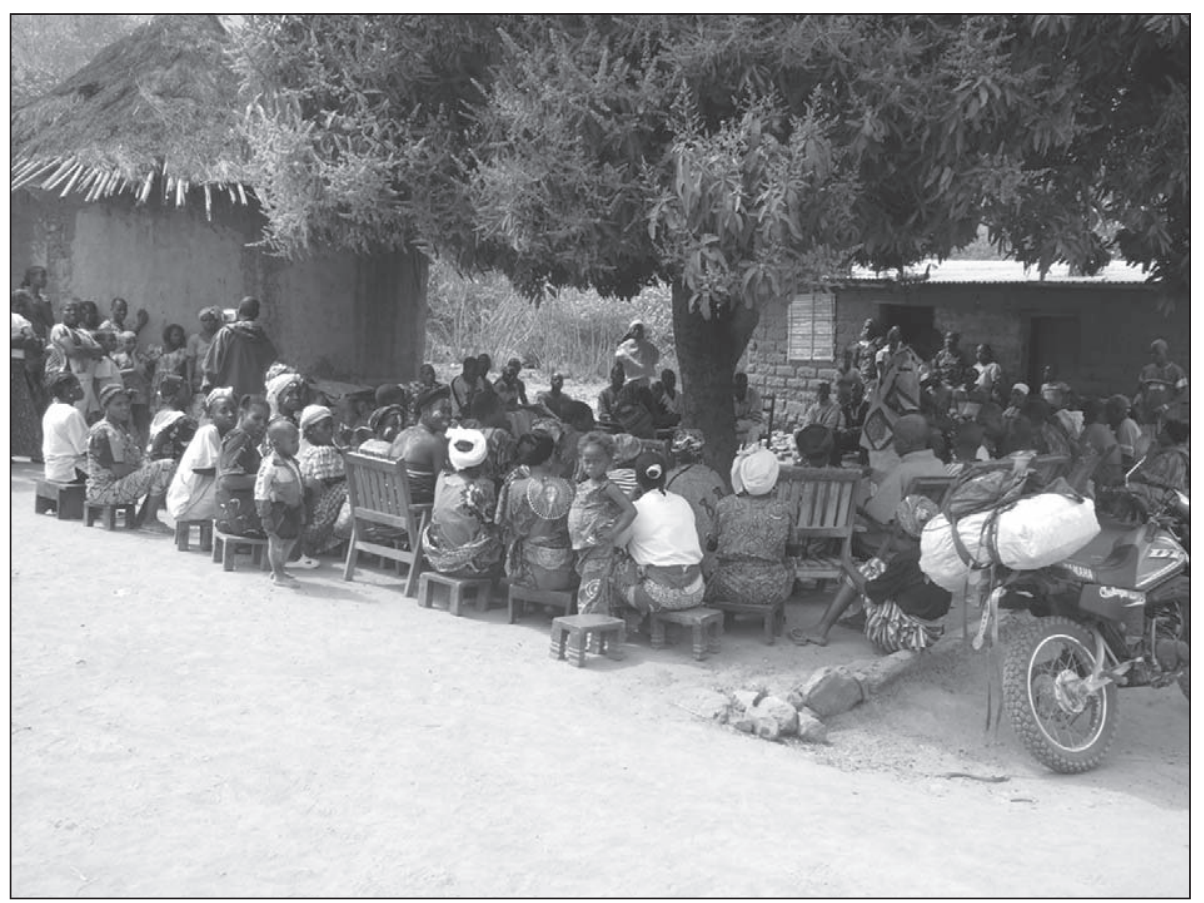

Figure 4. Assemblée du Kono, à Ditomba, le 8 janvier 2007.

Figure 4. A Kono meeting, Ditomba, January 8, 2007. 
mique comme facteur susceptible de motiver l'adhésion. Plutôt que de marginaliser les aînés et les notables - supposés réfractaires à l'innovation - tout le poids fut donné à leur avis dans la phase décisive des négociations conduisant au renoncement à leur terre ancestrale et à sa réappropriation au travers de son nouveau statut de parc. Le bouleversement que cela impliquait pour la société locale pouvait se préparer dans le respect des prérogatives sociales reconnues par tous. Conséquence remarquable de ce choix stratégique: ce sont les aînés et les notables dits "illettrés", et non pas l'"élite" francophone, qui, dans la période de crise et de remise en question du parc, se révèlent être les avocats les plus éloquents et les plus écoutés de sa sauvegarde et de sa réhabilitation.

Le message de réappropriation du parc, négocié dès 2006 au sein du Codiv, puis validé au travers de sa mise en circuit parmi la population et ses dirigeants, ne s'éloigne guère, par sa teneur, des objectifs initialement fixés par l'administration. Excluant toute velléité de récupération des droits ancestraux sur le parc, il met cependant l'accent sur une participation réelle aux activités visant sa sauvegarde et son exploitation touristique.

Si l'enquête LAGSUS a été créditée d'un rôle de catalyseur dans la relance du débat au sujet du parc, l'assimilant à la main proverbiale intervenant du côté du dos, il convient de porter au seul crédit des acteurs locaux la part symbolisée par la main occupée à laver le ventre. En effet : - la réhabilitation du parc comme propriété à sauvegarder et à valoriser s'effectue sans la moindre ingérence externe, exclue d'emblée par le double handicap de l'enclavement en zone rebelle et l'isolement géographique des lieux ;
- dans la chronologie du conflit ivoirien, cette relance endogène du parc s'amorce au moins une année avant les accords de Ouagadougou de mars 2007 qui marquent la fin officielle de la guerre, donc bien avant le début de ce qu'il sera convenu d'appeler la "sortie de crise ".

\section{Conclusion}

Imposé au départ, le message du PNMS est reconstitué spontanément, dans des conditions de rupture radicale avec sa source d'origine, au sein de la société à laquelle il avait été destiné, offrant ainsi un exemple parfait de "durabilité communicationnelle " (LAGSUS, 2009). Si celle-ci ne se confond pas avec la durabilité du parc en tant qu'objet de conservation, elle en constitue cependant, à l'heure actuelle, un des atouts majeurs. Loin d'être l'effet d'un simple retour à un état antérieur à la crise, elle est plutôt issue de celle-ci. L'acquis dynamique d'une prise de conscience spontanée des gens concernant la valeur de la conservation incarnée dans "leur parc ", s'il est de bon augure, non seulement pour l'avenir de ce dernier, mais aussi en tant qu'exemple d'une maîtrise des enjeux écologiques par une population qui, bien que située en dehors des grands circuits économiques, est stratégiquement placée par rapport à l'équilibre écologique d'une région dont l'étendue dépasse ce cadre restreint. Il faut espérer que, dans l'intérêt de la sauvegarde du PNMS en tant qu'écosystème d'importance régionale et nationale, cet acquis soit reconnu et intégré dans la feuille de route pour un meilleur après-guerre du parc et des habitants de sa zone périphérique.

\section{Références}

Alvarez MD. Forests in the Time of Violence. Conservation Implications of the Colombian War. Journal of Sustainable Forestry 2003; 16 : $1-20$.

Baya J. La communication en temps de crise dans un projet de gestion de ressources naturelles. In: Tourneux $\mathrm{H}$, ed. Langues, cultures et développement. Paris : Karthala, 2008.

Bearth T. Language as a key to understanding development from a local perspective. A case study from Ivory Coast. In: Tourneux $\mathrm{H}$, ed. Langues, cultures et développement. Paris: Karthala, 2008.

Bearth T, Fan D. The local language - a neglected resource for sustainable development. In : HessLüttich EWB, ed. Eco-Semiotics. Umwelt- und Entwicklungskommunikation. France : Tubingue, 2006.

ENGREF. (= École nationale du génie rural, des eaux et des forêts). Parc national du Mont Sangbé. Contribution à l'étude du parc et de sa zone périphérique. Montpellier : Engref, 1996.

Guéneau S, Jacobée F. Conservation de la biodiversité forestière tropicale en Afrique centrale. dépassionner les débats. Idées pour le débat, $n^{\circ} 14$. Paris: Institut du développement durable et des relations internationales, 2005.

Halle B, Bruzon F. Profil Environnemental de la Côte d'lvoire. Rapport final. Bruxelles: Commission européenne, 2006

Ministère de l'Environnement, de l'Eau et de la Forêt (MEEF). Aménagement du Parc national du MONT SANGBÉ et développement de sa périphérie. Tomes 1-2. Abidjan: MEEF, Direction de la protection de la nature, 2000-2001.

Ouattara S, Patard F, Brossard S. Projet de gestion de terroirs villageois et de développement local de deux zones périphériques du Parc National du Mont Sangbé. Rapport de fin de contrat mars 1995-2001. Abidjan: AFVP, Délégation en République de Côte d'Ivoire, 2001.

Price SV War and Tropical Forests. Conservation in Areas of Armed Conflict. New York : Haworth, 2003. 